\title{
Pathogenicity characterization with implicit and explicit molecular dynamics simulation
}

\author{
Sigit Jaya Herlambang ${ }^{*}$, Rosari Saleh \\ Department of Physics, Mathematics and Science Faculty, Universitas Indonesia, Depok, Indonesia; \\ *Corresponding Author: sigit.jaya.herlambang@gmail.com
}

Received 3 October 2011; revised 8 November 2011; accepted 19 November 2011.

\begin{abstract}
The contribution of water molecules in molecular dynamics simulation (MDS) is unquestionably high, particularly for enzymatic interaction which occurred in the cytoplasmic environment. The addition of water molecules to the system will surely influence different direct interaction between active site residues and substrate. We try to theoretically investigate to what extent the pathogenicity characterization will varies in different neuraminidase-sialic acid complex systems. The heating dynamics simulations were produced with and without TIP3P water molecules. The periodic boundary system was made for explicitly added TIP3P water molecules and generalized born molecular volume (GBMV) energy contribution was added for implicit solvent system. Both complexes, neuraminidase-sialic acid of $A / T o k y o / 3 / 67$ and $A / P e n n s y l v a n i a / 10218 /$ 84 , which have a different pathogenicity levels were minimized and simulated. The result shows more residues produced hydrogen bonds with substrate when water molecules were not added to the system. The binding free energies also show differences. Overall, even the values of energy is different, but an implicit solvent provides the similar result (HPAl complex has higher activity than LPAI for both systems) in characterization of pathogenic virus neuraminidase activity.
\end{abstract}

Keywords: Implicit; Explicit; Solvation; Molecular Dynamics Simulation; Neuraminidase; Sialic Acid

\section{INTRODUCTION}

The correlation between neuraminidase (NA) catalytic activity with the pathogenicity level has been studied in several experiments [1-11]. The results shows the same similarity that the high pathogenic avian influenza (HPAI) virus have NA's activity higher than the low pathogenic avian influenza (LPAI). Further investigation even revealed specific NA's residue which increase the virulence, viral pathogenicity, and cause a resistance into NA inhibitors (NAI).

The NA catalytic process, which cleavage matured virion that attached in the cell wall, occurred in the aqueous environment. To produce molecular dynamics simulation of NA-SA complex, the contribution of water molecules to the interactions need to be added into the system. Since, the addition of water molecules explicitly into the system requires high computational costs, many alternative methods were achieved to add aqueous environment implicitly to suppress the time of simulation. One of many methods widely used is GBMV [12].

In this study, the NA-SA complexes were compared in explicit and implicit solvent to get insight on how the results still have a capability to characterizing the pathogenicity level of avian influenza virus. The comparison was build in the subject of the structural changes through root mean square deviation (RMSD), the number of NA residues formed hydrogen bond with SA directly and indirectly, percentage of occupation, and free binding energy.

\section{MATERIALS AND METHODS}

\subsection{Structure Preparation}

The NA sequence of A/Tokyo/3/67 avian influenza virus (AIV), that was isolated in 1967 [13] during a time of prevalent infection, obtained from NCBI [14] with accession code AAB05621. The sequence was being aligned with the NA sequence of A/Pennsylvania/10218/ 84 (accession code BAF48360) which is a non-pathogenic AIV [15]. These AIV's were chosen to delegated two different level of pathogenicity. Sequences alignment was executed with BLOSUM 30 scoring matrix in fast pairwise alignment method. 
The result of sequences alignment was being used in homology modeling to generate LPAI NA molecule. The template molecule, which is A/Tokyo/3/67 NA-SA complex [16], was obtained from RCSB protein data bank [17]. The CHARMm forcefield utilized to adding missing hydrogen atoms $[18,19]$. The original LPAI and HPAI NA-SA complex molecules were copied and the explicit water molecules were added into duplicated molecules as TIP3P waterbox [20].

\subsection{Minimization and Heating MDS}

The minimization and heating MDS were done with periodic boundary implicit and explicit solvation systems. The GBMV implicit solvation was included in original and unsolvated HPAI and LPAI NA-SA complexes minimization and heating MDS. Both HPAI and LPAI complexes in both systems minimized within two steps, 1,000,000-steps maximum with gradient energy $0.5 \mathrm{kcal} / \mathrm{mol}$ steepest descents and 1,000,000-steps maximum with gradient energy $0.1 \mathrm{kcal} / \mathrm{mol}$ conjugate gradient. Heating MDS was successfully completed with 20 picoseconds (ps) time simulation. All systems temperature rose from 0 into $300 \mathrm{~K}$ with the parameters used were 20,000 steps, 0.001 time step, non-bond list radius $14 \AA$, switching function 10-12 $\AA[21,22]$, the SHAKE algorithm [23] was applied (only in the implicit solvation) to fix lengths of all bonds involving hydrogen atoms, and the trajectory data were stored every $0.1 \mathrm{ps}$. All phases of the study described in this section from structure preparation to molecular dynamics simulation were conducted with Discovery Studio 2.1 (Accelrys).

\section{RESULTS}

\subsection{All Atoms, Backbone, and SA RMSD}

Figures 1(a), 1(b), and 1(c) visualize the structural changes during the simulation. The movement of subjected atoms could be monitored through its fluctuative RMSD. The higher fluctuate indicates higher movement. The structural stability of each molecule and the whole atoms in the complexes could also be analyzed from those figures. Overall, the explicitly solvated complexes show much better stability than the implicit.

Figure 1(a) depicts the RMSD of all atoms in the complex (without water molecules). Both solvation shows progressive increasement around $0-5 \mathrm{ps}$, but in the implicit solvation systems, the movements of all atoms are higher than in the explicit. This difference must be caused by the water molecules that surrounded the NA-SA complexes. Although the stability of implicit solvation systems is lower than the other, it has an advantage. A significant result in pathogenicity characterization is more

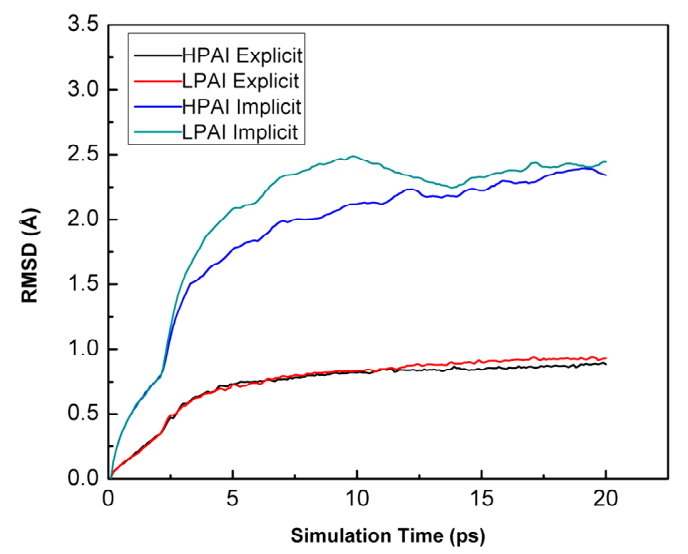

(a)

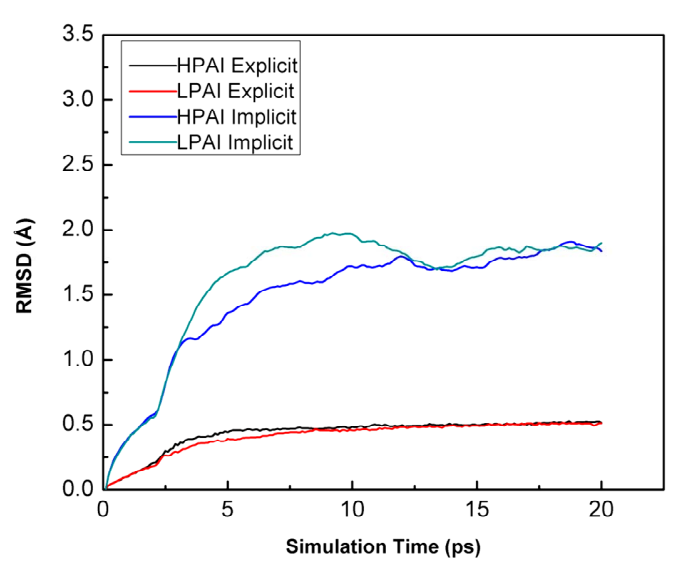

(b)

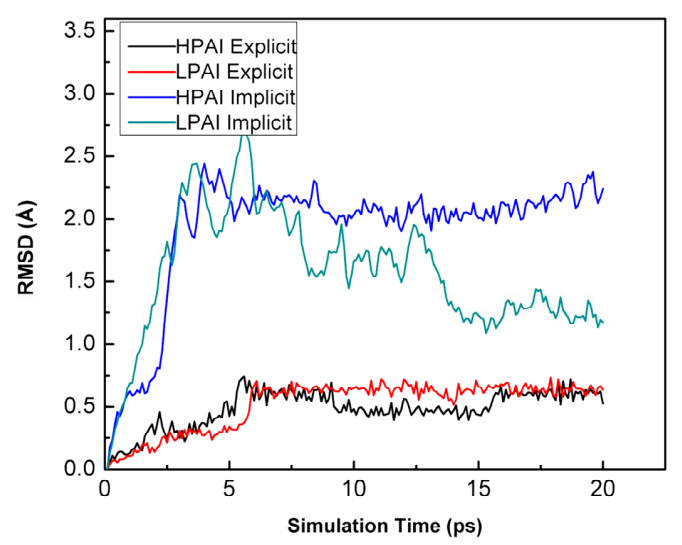

(c)

Figure 1. The RMSD vs simulation time of: (a) NA-SA complexes all atoms; (b) NA backbone atoms; (c) SA atoms.

obvious since the LPAI shows a higher movement during the simulation. Similar result in the pathogenicity characterization showed in Figure 1(b) which differed only on the value of the RMSD. The value of all atoms RMSD is higher than the NA backbone.

Figure 1(c) shows SA movement during the simula- 
tion. With explicit solvation, SA which is bound in the HPAI NA binding pocket RMSD fluctuate more than in LPAI NA. The fluctuation noticed around $10-15$ ps in simulation time. The result shows differences in the implicit solvation which have higher fluctuation of SA RMSD when bound the LPAI NA than in the HPAI NA. Different interaction in the explicit solvation between SA-water molecules may cause the different SA movement in the NA binding pocket during the simulation.

\subsection{The Hydrogen Bonds between NA Residues and SA}

The comparison of hydrogen bonds formed between functional residues (residues which interact directly with substrate, in this case is SA) and SA has been done. This is needed since hydrogen bonds cluster bind tightly. Table 1 listed all NA residues that formed hydrogen bonds with SA complemented with its interaction energy and distance. This section also provided the percentage of hydrogen bonds occupation and the figure of water molecules in explicit solvation which linkage framework residues to bind SA indirectly.

In Table 1 the results shows that the HPAI NA-SA complex produce larger number of hydrogen bonds formed, compared with LPAI NA-SA complex, in the last conformation. This is appears in both implicit and explicit solvation systems. In implicit HPAI NA-SA complex there are 15 hydrogen bonds formed while LPAI only have 8 . The best interaction (lowest interaction energy) and the shortest distance of NA residues-SA in an implicit solvation was made by hydrogen bond formed between $\operatorname{Arg} 152 \mathrm{HH} 21$ atom and SA O7 atom in the HPAI complex with $-41.61 \mathrm{kcal} / \mathrm{mol}$ and $1.81 \AA$, respectively.

In explicit LPAI NA-SA complex there are only 4 hydrogen bonds formed while in HPAI produced 7 hydrogen bonds. The lowest interaction energy was made by hydrogen bond formed between Glu276 OE1 atom and SA H5 atom with $-41.11 \mathrm{kcal} / \mathrm{mol}$. The shortest distance was made by hydrogen bond formed between Asp151 OD1 atom and SA $\mathrm{H} 3$ atom with $1.73 \AA$. In the last conformation of simulation also revealed several water molecules formed hydrogen bond with the SA. Fascinatingly, the water molecules which linkage several residues appears only in HPAI (look Figures 2(a) and 2(b)). Those residues are Glu119, Asp151, Ser179, Arg224, and Glu227.

The percentage of occupation offers a complementary observation into the stability of hydrogen bonds formed during the simulation. From Figure 3 could be seen that several functional residues formed hydrogen bonds with SA which have percentage of occupation larger than $80 \%$. This is indicated that those residues have strong hydrogen bonds [23]. In the explicit HPAI NA-SA complex observed that there are four NA residues formed a strong hydrogen bond with SA. Those residues are Asp151, Arg152, Glu276, and Arg371. In other hand, the explicit LPAI NA-SA complex produce three strong hydrogen bonds that formed by Asp151, Arg224, and Glu276 with SA atoms.

In the implicit HPAI NA-SA complex there are produced five strong hydrogen bonds formed by SA with Arg118, Arg152, Glu276, Arg292, and Arg371. Three of those five residues are known as triad Arg 118-292-371 which occupy S1 active site. This is not occurred in the implicit LPAI NA-SA complex which formed four strong hydrogen bonds involving Asp151, Glu276, Arg292, and Tyr406. In spite of that, the results shows similarity that the numbers of strong hydrogen bonds formed in HPAI is larger than LPAI in both solvation applied in NA-SA complexes for the simulation. For both solvation also revealed that strong hydrogen bonds was made by Glu276 for all complexes simulated. This indicates that the Glu276 has played a role in NA-SA binding.

\subsection{End-Point Free Binding Energy}

Table 2 shows free binding energies of all NA-SA complex molecules in the last conformation based on Amaro et al. [24]. This method improves the time of calculation than if all of trajectories saved were calculated. The results are known similar to other studies that calculated the binding free energy of NA-inhibitor complexes with different approaches $[25,26]$. In the explicit solvation systems, complex molecules calculated without any implicit solvation energetic contribution while in the implicit solvation, the calculation executed with the addition of GBMV implicit solvation energy. The NASA complexes in HPAI is lower than LPAI for both solvation which in other way tells that the HPAI NA have a better interaction with SA than in the LPAI [27,28]. The similarity of the results could be seen as the indicator that pathogenicity characterization is available to perform either with implicit solvation or explicit solvation.

\section{DISCUSSION}

The main focus in this study is to compare the implicit solvation GBMV with explicit solvation and both possibility to determine the difference of pathogenicity of AIV through the interaction of its NA-SA. Overall, almost all of the results show similarities. Except in the SA RMSD that shown different stability between HPAI and LPAI in both implicit and explicit solvation. In the implicit solvation the HPAI is more stable than LPAI while in explicit solvation the LPAI is the more stable than HPAI. Even in the explicit solvation the differences 
Table 1. The hydrogen bonds formed in the last of simulation completed with its energy of interaction and distance.

\begin{tabular}{|c|c|c|c|c|}
\hline \multicolumn{5}{|c|}{ Hydrogen Bonds } \\
\hline Atom 1 & Atom 2 & E (kcal/mol) & Distance ( $\AA$ ) & \\
\hline Arg152 HH21 & Sialic Acid O10 & -36.42 & 2.45 & Explicit HPAI \\
\hline Arg292 НH22 & Sialic Acid O1B & -28.52 & 2.13 & \\
\hline Arg371 HH12 & Sialic Acid O1A & -32.84 & 2.22 & \\
\hline Arg371 HH12 & Sialic Acid O1B & -31.47 & 2.03 & \\
\hline Arg371 HH22 & Sialic Acid O1B & -34.45 & 2.33 & \\
\hline Asp151 OD1 & Sialic Acid H3 & -34.24 & 1.73 & \\
\hline Glu276 OE1 & Sialic Acid H5 & -41.11 & 1.90 & \\
\hline Asp151 OD1 & Sialic Acid H15 & -39.43 & 2.29 & Explicit LPAI \\
\hline Arg224 HH11 & Sialic Acid O9 & -36.62 & 2.44 & \\
\hline Arg371 HH22 & Sialic Acid O1A & -30.54 & 2.07 & \\
\hline Glu276 OE1 & Sialic Acid H17 & -32.79 & 2.01 & \\
\hline Arg118 HH12 & Sialic Acid O4 & -37.87 & 2.00 & Implicit HPAI \\
\hline $\operatorname{Arg} 152 \mathrm{HE}$ & Sialic Acid O10 & -24.59 & 2.24 & \\
\hline Arg152 HH21 & Sialic Acid O7 & -41.61 & 1.81 & \\
\hline Arg224 HH11 & Sialic Acid O9 & -32.64 & 2.32 & \\
\hline Arg292 HH22 & Sialic Acid O1B & -35.73 & 1.95 & \\
\hline Arg292 HН22 & Sialic Acid O1A & -28.50 & 2.45 & \\
\hline Arg371 HH12 & Sialic Acid O1B & -35.91 & 1.94 & \\
\hline Arg371 HH22 & Sialic Acid O1A & -28.82 & 2.43 & \\
\hline Arg371 HH22 & Sialic Acid O1B & -37.08 & 1.86 & \\
\hline Tyr406 OH & Sialic Acid H1 & -27.82 & 1.91 & \\
\hline Glu119 OE2 & Sialic Acid H3 & -40.74 & 1.92 & \\
\hline Glu276 OE1 & Sialic Acid H5 & -41.52 & 1.87 & \\
\hline Glu276 OE2 & Sialic Acid H5 & -33.75 & 2.37 & \\
\hline Glu276 OE1 & Sialic Acid H6 & -34.78 & 2.30 & \\
\hline Glu276 OE2 & Sialic Acid H6 & -34.57 & 2.31 & \\
\hline Arg292 HH12 & Sialic Acid O1A & -31.79 & 2.20 & Implicit LPAI \\
\hline Arg292 HH12 & Sialic Acid O1B & -36.55 & 1.90 & \\
\hline Arg292 HH21 & Sialic Acid O8 & -33.60 & 2.25 & \\
\hline Arg292 HH22 & Sialic Acid O1A & -36.09 & 1.93 & \\
\hline Tyr406 HH & Sialic Acid O1B & -41.15 & 1.90 & \\
\hline Asp151 OD1 & Sialic Acid H15 & -39.57 & 2.00 & \\
\hline Asp151 OD2 & Sialic Acid H15 & -37.91 & 2.10 & \\
\hline Glu276 OE2 & Sialic Acid H17 & -41.31 & 1.88 & \\
\hline
\end{tabular}




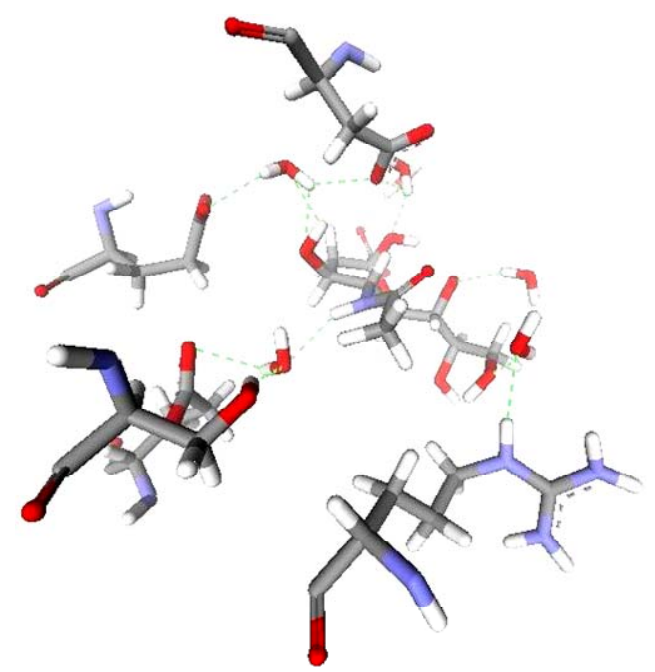

(a)

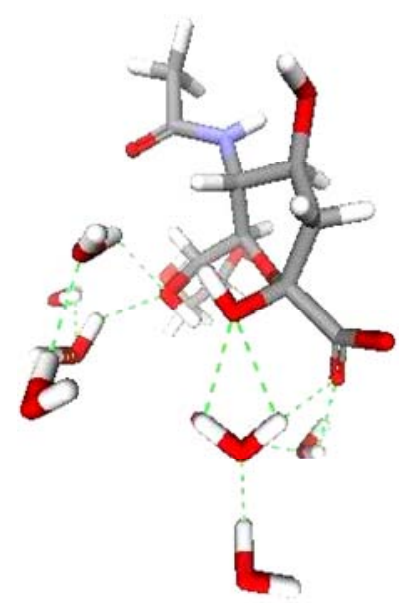

(b)

Figure 2. The water molecules which produce hydrogen bond with SA in: (a) HPAI NA-SA complex; (b) LPAI NA-SA complex.

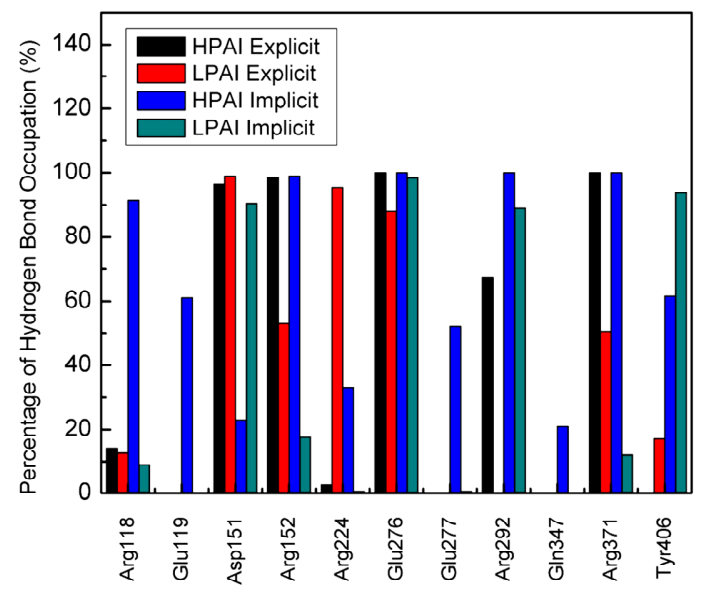

Figure 3. The percentage occupation of hydrogen bonds formed during the simulation.
Table 2. Free binding energy.

\begin{tabular}{cccc}
\hline \multicolumn{4}{c}{ Free Energy $(\mathrm{kcal} / \mathrm{mol})$} \\
\hline $\begin{array}{c}\text { HPAI Explicit } \\
\text { Solvation }\end{array}$ & $\begin{array}{c}\text { LPAI Explicit } \\
\text { Solvation }\end{array}$ & $\begin{array}{c}\text { HPAI Implicit } \\
\text { Solvation }\end{array}$ & $\begin{array}{c}\text { LPAI Implicit } \\
\text { Solvation }\end{array}$ \\
-361.55 & -273.64 & -108.60 & 2.85 \\
\hline
\end{tabular}

is subtle, it could still be an indicator that there is may be another explanation for this confusing results. It is probably be caused by the interaction of SA with water molecules in explicit solvation simulation. As indirect interaction was made in HPAI from the residues that formed a hydrogen bonds with water molecules that also produces a hydrogen bonds with SA, which did not occurred in LPAI NA-SA complex. However, deep investigation is still needed to look further in this issue.

The NA-SA binding with and without water molecules influences different positional fluctuations of complex molecules. It appears in our RMSD graphics that the molecules in the explicit solvation system have lower RMSD values. Non-bond interactions between complex molecules and water molecules stabilize the system that cause the movement of the complex molecules is minimum. In both explicit and implicit solvation, HPAI has more stable movement which can be seen from its minimum fluctuation RMSD. This RMSD comparison is amenable with multiple studies that infer that a higher substrate RMSD suggests the superior neuraminidase ability to reject an inhibitor $[23,29,30]$. While in this study, SA high RMSD related with neuraminidase acceptance abilities when cleavage the virion from the edge of the cell.

The specific residues that formed hydrogen bonds with SA in our experiment agree with the results from other studies [1-11]. The key role of Asp151, Glu276, $\operatorname{Arg} 292$, and Arg371 are important that the changes in one of those residues could followed by the resistance of inhibitors. In both explicit and implicit solvation systems, their importance is obviously observed. It could be seen from its interaction energy, distance and percentage of occupation. Those three results provide the evidence that they have a strong interaction in almost all NA-SA complexes simulated.

The end point free binding energy calculation results for explicit simulation is very low. It seems because the calculation for the explicit solvation systems, the implicit solvation contribution was not added. The GBMV free binding energy calculation then also executed for explicit complex molecules. The results show the values of $-1.37 \mathrm{kcal} / \mathrm{mol}$ and $17.97 \mathrm{kcal} / \mathrm{mol}$ for HPAI and LPAI, respectively. Compared by other studies $[25,26$, 31], the calculation with GBMV is at their results range $(-21.7 \mathrm{kcal} / \mathrm{mol}$ to $7.3 \mathrm{kcal} / \mathrm{mol})$. This is indicate that our 
results could be used to characterize virus pathogenicity.

\section{CONCLUSION}

Differences in pathogenicity level can be assessed by implicit or explicit solvation heating dynamics simulation. Even the structural changes and energetic analysis show significant value, the implicit solvation systems results are similar to with the explicit solvation systems. The HPAI complexes molecules in both systems show higher activity than the LPAI complexes molecules. The use of explicit solvation is suggested for better results and systems stability, but the use of implicit solvation is still resembles the explicit solvation.

\section{ACKNOWLEDGEMENTS}

We would like to express gratitude towards Ding Ming Chee of Accelrys Singapore for the Accelrys Discovery Studio 2.1 trial sent to us.

\section{REFERENCES}

[1] Russell, R.J., Haire, L.F., Stevens, D.J., Collins, P.J., Lin, Y.P., Blackburn, G.M., Hay, A.J., Gamblin, S.J. and Skehel, J.J. (2006) The structure of H5N1 avian influenza neuraminidase suggests new opportunities for drug design. Nature, 443, 45-49. doi:10.1038/nature05114

[2] Chachra, R. and Rizzo, R.C. (2008) Origins of resistance conferred by the R292K neuraminidase mutation via molecular dynamics and free energy calculations. Journal of Chemical Theory and Computation, 4, 1526-1540. doi:10.1021/ct800068v

[3] McKimm-Breschkin, J.L., Sahasrabudhe, A., Blick, T.J., McDonald, M., Colman, P.M., Hart, G.J., Bethell, R.C. and Varghese, J.N. (1998) Mutations in a conserved residue in the influenza virus neuraminidase active site decreases sensitivity to Neu5Ac2en derivatives. Journal of Virology, 72, 2456-2462.

[4] Mishin, V.P., Hayden, F.G. and Gubareva, L.V. (2005) Susceptibilities of antiviral-resistant influenza viruses to novel neuraminidase inhibitors. Antimicrobial Agents and Chemotherapy, 49, 4515-4520.

doi:10.1128/AAC.49.11.4515-4520.2005

[5] Sheu, T.G., Deyde, V.M., Okomo-Adhiambo, M., Garten, R.J., Xu, X., Bright, R.A., Butler, E.N., Wallis, T.R., Klimov, A.I. and Gubareva, L.V. (2008) Surveillance for neuraminidase inhibitor resistance among human influenza A and B viruses circulating worldwide from 2004 to 2008. Antimicrobial Agents and Chemotherapy, 52, 32843292.

[6] Wetherall, N.T., Trivedi, T., Zeller, J., Hodges-Savola, C., McKimm-Breschkin, J.L., Zambon, M. and Hayden, F.G. (2003) Evaluation of neuraminidase enzyme assays using different substrates to measure susceptibility of influenza clinical isolates to neuraminidase inhibitors: Report of the Neuraminidase Inhibitor Susceptibility Network. Journal of Clinical Microbiology, 41, 742-750. doi:10.1128/JCM.41.2.742-750.2003

[7] McKimm-Breschkin, J.L., Trivedi, T., Hampson, A., Hay, A., Klimov, A., Tashiro, M., Hayden, F.G. and Zambon,
M. (2003) Neuraminidase sequence analysis and susceptibilities of influenza virus clinical isolates to zanamivir and oseltamivir. Antimicrobial Agents and Chemotherapy, 47, 2264-2272. doi:10.1128/AAC.47.7.2264-2272.2003

[8] Yen, H., Ilyushina, N.A., Salomon, R., Hoffmann, E., Webster, R.G. and Govorkova, E.A. (2007) Neuraminidase inhibitor-resistant recombinant a/vietnam/1203/04 (H5N1) influenza viruses retain their replication efficiency and pathogenicity in vitro and in vivo. Journal of Virology, 81, 12418-12426. doi:10.1128/JVI.01067-07

[9] Meijer, A., Lackenby, A., Hungnes, O., Lina, B., van der Werf, S., Schweiger, B., Opp, M., Paget, J., van de Kassteele, J., Hay, J. and Zambon. M. (2009) Oseltamivir-resistant influenza virus A (H1N1), Europe, 200708 Season. Emerging Infectious Diseases, 15, 552-560. doi:10.3201/eid1504.081280

[10] Monto, A.S., McKimm-Breschkin, J.L., Macken, C., Hampson, A.W., Hay, A., Klimov, A., Tashiro, M., Webster, R.G., Aymard, M., Hayden, F.G. and Zambon, M. (2006) Detection of influenza viruses resistant to neuralminidase inhibitors in global surveillance during the first 3 years of their use. Antimicrobial Agents and Chemotherapy, 50, 2395-2402. doi:10.1128/AAC.01339-05

[11] Tamura, D., Mitamura, K., Yamazaki, M., Fujino, M., Nirasawa, M., Kimura, K., Kiso, M., Shimizu, H., Kawakami, C., Hiroi, S., Takahashi, S., Hata, M., Minagawa, H., Kimura, Y., Kaneda, S., Sugita, S., Horimoto, T., Sugaya, N. and Kawaoka, Y. (2009) Oseltamivir-resistant influenza A viruses circulating in Japan. Journal of Clinical Microbiology, 47, 1424-1427. doi:10.1128/JCM.02396-08

[12] Lee, M.S., Feig, M., Salsbury, F. R. and Brooks, C.L.III. (2003) New analytic approximation to the standard molecular volume definition and its application to generalized born calculations. Journal of Computational Chemistry, 24, 1348-1356. doi:10.1002/jcc. 10272

[13] Pereira, M.S., Chakraverty, P. and Pane, A.R. (1969) The influence of antigenic variation on influenza A2 epidemics. Journal of Hygiene, 67, 551-557.

[14] http://www.ncbi.nlm.nih.gov/genomes/FLU/Database/np h-select.cgi?go=database

[15] Kaverin, N.V., Rudneva, I.A., Ilyushina, N.A., Varich, N.L., Lipatov, A.S., Smirnov, Y.A., Govorkova, E.A., Gitelman, A.K., Lvov, D.K. and Webster, R.G. (2002) Structure of antigenic sites on the haemagglutinin molecule of $\mathrm{H} 5$ avian influenza virus and phenotypic variation of escape mutants. Journal of General Virology, 83, 2497-2505.

[16] Varghese, J.N., Mc-Kimm-Breschkin, J.L., Caldwell, J.B., Kortt, A.A. and Colman, P.M., (1992) The structure of the complex between influenza virus neuraminidase and sialic acid, the viral receptor. Proteins, 14, 327-332. doi: $10.1002 /$ prot. 340140302

[17] http://www.rcsb.org/pdb/home/home.do

[18] Brooks, B.R., Bruccoleri, R.E., Olafson, B.D., States, D.J., Swaminathan, S. and Karplus, M. (1983) CHARMM: A program for macromolecular energy minimization and dynamics calculations. Journal of Computational Chemistry, 4, 187-217. doi:10.1002/jcc.540040211

[19] Brooks, B.R., et al., (2009) CHARMM: The biomolecular simulation program. Journal of Computational Chemistry, 30, 1545-1614. doi: $10.1002 /$ jcc. 21287 
[20] Jorgensen, W.L., Chandrasekhar, J., Madura, J.D., Impey, R.W. and Klein, M.L. (1983) Comparison of simple potential functions for simulating liquid water. Journal of Chemical Physics, 79, 926-935. doi:10.1063/1.445869

[21] Brooks, C.L.III, Pettit, B.M. and Karplus, M. (1985) Structural and energetic effects of truncating long ranged interactions in ionic and polar fluids. Journal of Chemical Physics, 83, 5897-5908. doi:10.1063/1.449621

[22] Steinbach, P.J. and Brooks, B.R. (1994) New spherecal-cutoff methods for long-range forces in macromolecular simulation. Journal of Computational Chemistry, 15, 667-683. doi:10.1002/jcc.540150702

[23] Shu, M., Lin, Z., Zhang, Y., Wu, Y., Mei, H. and Jiang, Y. (2010) Molecular dynamics simulation of oseltamivir resistance in neuraminidase of avian influennza H5N1 virus. Journal of Molecular Modeling, 17, 587-592.

[24] Amaro, R.E., Cheng, X., Ivanov, I., Xu, D. and McCammon, J.A. (2009) Characterizing loop dynamics and ligand recognition in human- and avian-type influenza neuraminidases via generalized born molecular dynamics and end-point free energy calculations. Journal of the American Chemical Society, 13, 4702-4709. doi:10.1021/ja8085643

[25] Masukawa, K.M., Kollman, P.A. and Kuntz, I.D. (2003) Investigation of neuraminidase-substrate recognition using molecular dynamics and free energy calculations. Journal of Medicinal Chemistry, 46, 5628-5637. doi:10.1021/jm030060q

[26] Smith, B.J., Colman, P.M., von Itzstein, M., Danylec, B. and Varghese, J.N. (2000) Analysis of inhibitor binding in influenza virus neuraminidase. Protein Science, 10,
689-696. doi:10.1110/ps.41801

[27] Guo, X.L., Wei, D.Q., Zhul, Y.S. and Chou, K.C. (2008) Cleavage mechanism of the $\mathrm{H} 5 \mathrm{~N} 1$ hemagglutinin by trypsin and furin. Amino Acids, 35, 375-382.

[28] Decha, P., Rungrotmongkol, T., Intharathep, P., Malaisree, M., Aruksakunwong, O., Laohpongspaisan, C., Parasuk, V., Sompornpisut, P., Pianwanit, S., Kokpol, S. and Hannongbua, S. (2008) Source of high pathogenicity of an avian influenza virus H5N1: Why H5 is better cleaved by furin. Biophysical Journal, 95, 128-134. doi:10.1529/biophysj.107.127456

[29] Aruksakunwong, O., Malisree, M., Decha, P., Sompornpisut, P., Parasuk, V., Pianwanit, S. and Hannongbua, S. (2007) On the lower susceptibility of oseltamivir to influenza neuraminidase subtype than those in N2 and N9. Biophysical Journal, 92, 798-807. doi:10.1529/biophysj.106.092528

[30] Le, L., Lee, E., Schulten, K. and Truong, T.N. (2009) Molecular modeling of swine influenza A/H1N1, Spanish $\mathrm{H} 1 \mathrm{~N} 1$, and avian H5N1 flu N1 neuraminidases bound to tamiflu and relenza. PloS Current. http://www.ncbi.nlm.nih.gov/pmc/articles/PMC2762416. 4/

[31] von Itzstein, M., Wu, W.Y., Kok, G.B., Pegg, M.S., Dyason, J.C., Jin, B., Phan, T.V., Smythe, M.L., White, H.F., Oliver, S.W., Colman, P.M., Varghese, J.N., Ryan, D.M., Woods, J.M., Bethell, R.C., Hotham, V.J., Cameron, J.M. and Penn, C.R. (1993) Rational design of potent sialidase-based inhibitors of influenza virus replication. Nature, 363, 418-423. doi:10.1038/363418a0

\section{ABBREVIATION}

\begin{tabular}{clllll}
\hline Gly & Glycine & Met & Methionine & Trp & Tryphtophan \\
Ala & Alanine & Cys & Cysteine & Glu & Glutamic Acid \\
Ile & Isolucine & Asn & Asparagine & His & Histidine \\
Leu & Leucine & Gln & Glutamine & Lys & Lysine \\
Val & Valine & Ser & Serine & Phe & Phenylalanine \\
Pro & Proline & Thr & Threonine & Asp & Aspartat Acid \\
Tyr & Tyrosine & Arg & Arginine & & \\
\hline
\end{tabular}

